\title{
AN INEQUALITY FOR FUNCTIONS WITH DERIVATIVES IN AN ORLICZ SPACE
}

\author{
R. BOJANIC AND J. MUSIELAK
}

The purpose of this note is to give a simple proof of an inequality for differentiable functions of several variables. This inequality is an extension of a well-known inequality of Sobolev and it is used to simplify the proofs of certain results from the theory of distributions.

1. In the following we shall assume that $M(u)$ is an even, convex and continuous function defined for all real $u$, such that $M(0)=0$, $M(u)>0$ for $u \neq 0, M(u) / u \rightarrow 0 \quad(u \rightarrow 0)$ and $M(u) / u \rightarrow \infty \quad(u \rightarrow \infty)$. From these assumptions follows that there exists a nondecreasing function $p(v)$ defined for $v \geqq 0$, continuous from the right, satisfying conditions $p(0)=0, p(v) \rightarrow \infty(v \rightarrow \infty)$ and such that $M(u)=\int_{0}^{|u|} p(v) d v$ for all $u$. Writing $q(u)=\sup \{v: p(v) \leqq u\}$ and $N(u)=\int_{0}^{|u|} q(v) d v$, we have $q(p(v)) \geqq v$ and $q(p(v)-\epsilon) \leqq v$ for every $v \geqq 0$ and $\epsilon>0$ for which $p(v) \geqq \epsilon$. Moreover, the Young's ine yuality $u v \leqq M(u)+N(v)$ holds for all $u$ and $v$ (see $[1, \S \S 1-2]$ ).

Let $g$ be a real-valued, almost everywhere differentiable function. The proof of our main result is based on the following inequality

$$
(M(g(u)))^{\prime} \leqq M\left(g^{\prime}(u)\right)+M(2 g(u))
$$

which holds for almost all $u$. We have namely by Young's inequality

$$
(M(g(u)))^{\prime}=M^{\prime}(g(u)) g^{\prime}(u) \leqq M\left(g^{\prime}(u)\right)+N\left(M^{\prime}(g(u))\right)
$$

for almost all $u$. Thus we have only to prove that

$$
N\left(M^{\prime}(u)\right) \leqq M(2 u)
$$

for all $u$ for which the derivative $M^{\prime}(u)$ exists. Taking $u \neq 0$ and assuming that $M^{\prime}(u)$ exists we have for any $\epsilon>0$ such that $p(u) \geqq \epsilon$

$$
\begin{aligned}
N\left(M^{\prime}(u)-\epsilon\right) & =\int_{0}^{p(u)-\epsilon} q(v) d v \leqq(p(u)-\epsilon) q(p(u)-\epsilon) \\
& \leqq u p(u) \leqq \int_{u}^{2 u} p(v) d v \leqq M(2 u)
\end{aligned}
$$

and (2) follows by the continuity of $N(u)$.

Received by the editors July 30, 1963. 
2. In order to simplify the exposition wherever possible we shall use the following more or less generally accepted conventions.

The real $n$-dimensional Euclidean space will be denoted simply by $E$. For any $x=\left(x_{1}, \cdots, x_{n}\right) \in E$ we define $|x|$ by $|x|=\left|x_{1}\right|+\cdots$ $+\left|x_{n}\right|$.

If $f$ is a function defined in $E$ we shall use both notations $f(x)$ and $f\left(x_{1}, \cdots, x_{n}\right)$ to denote the value of $f$ at $x=\left(x_{1}, \cdots, x_{n}\right)$.

The following notation will be used for partial derivatives. Let $p_{i}, i=1, \cdots, n$ be nonnegative integers and $p=\left(p_{1}, \cdots, p_{n}\right)$. Then

$$
D^{p} f=\left(\partial^{|p|} / \partial x_{1}^{p_{1}} \cdots \partial x_{n}^{p_{n}}\right) f
$$

Also

$$
f_{i}^{\prime}=\left(\partial / \partial x_{i}\right) f, \quad i=1, \cdots, n .
$$

We shall denote by $P$ the set of all $p=\left(p_{1}, \cdots, p_{n}\right)$ such that $p_{i}=0$ or $p_{i}=1$ for $i=1, \cdots, n$. Thus $D^{p} f$ for $p \in P$ and $|p| \geqq 2$ is a mixed derivative of $f$ of order $|p|$.

Finally, if $g$ is an integrable real-valued function on a measurable $n$-dimensional subset $A$ of $E$, we shall denote the integral of $g$ over $A$ by $\int_{A} g(t) d t$.

Given a function $M$ satisfying conditions mentioned in $\$ 1$ and a measurable $n$-dimensional subset $A$ of $E$, the Orlicz space $L_{M}^{*}(A)$ is defined as the class of all real-valued measurable functions $f$ whose domain contains $A$, such that

$$
\int_{A} M(k f(t)) d t<\infty
$$

where $k>0$ is a constant depending only on $f$ and $A$. The space $L_{M}^{*}(A)$ where $f_{1}=f_{2}$ means that $f_{1}(t)=f_{2}(t)$ almost everywhere in $A$ is a Banach space with the norm

$$
\|f\|_{M}=\inf \left\{\epsilon>0: \int_{A} M\left(\epsilon^{-1} f(t)\right) d t \leqq 1\right\} .
$$

3. We shall denote by $C(x ; \rho)$ any one of the $2^{n} n$-dimensional closed cubes having a vertex at $x$ and edges of length $\rho$ parallel to the coordinate axes. We shall prove here the following theorem: then

If $f$ and its mixed derivatives $D^{p} f, p \in P$, are continuous in $C(x ; \rho)$

$$
M(f(x)) \leqq \sum_{p \in P}\left(1+\frac{1}{\rho}\right)^{n-|p|} \int_{C(x ; \rho)} M\left(2^{n-|p|} D^{p} f(t)\right) d t
$$


If we denote by $S(x ; R)$ the $n$-dimensional closed sphere with center at $x$ and of radius $R$, then $C(x ; R / \sqrt{ } n) \subset S(x ; R)$ and from the preceding theorem we obtain immediately the following extension of an inequality of L. Hörmander (see [2, p. 97]): then

If $f$ and its mixed derivatives $D^{p} f, p \in P$, are continuous in $S(x ; R)$

$$
M(f(x)) \leqq \sum_{p \in P}\left(1+\frac{\sqrt{ } n}{R}\right)^{n-|p|} \int_{S(x ; R)} M\left(2^{n-|p|} D^{p} f(t)\right) d t .
$$

Another consequence of our theorem is the following result:

If $f$ and its mixed derivatives $D^{p} f, p \in P$, are continuous in $\{t:|t| \geqq|x|\}$ then

$$
M(f(x)) \leqq \sum_{p \in P} \int_{|t| \geq|x|} M\left(2^{n-|p|} D^{p} f(t)\right) d t .
$$

To show this, let $x=\left(x_{1}, \cdots, x_{n}\right)$ and

$J_{k}=\left\{t_{k}:\left(x_{k} \leqq t_{k} \leqq x_{k}+\rho\right.\right.$ if $\left.x_{k} \geqq 0\right)$ or $\left(x_{k}-\rho \leqq t_{k} \leqq x_{k}\right.$ if $\left.\left.x_{k}<0\right)\right\}$.

Then it is sufficient to choose $C(x ; \rho)=J_{1} \times \cdots \times J_{n}$ and to observe that $C(x ; \rho) \subset\{t:|t| \geqq|x|\}$ for all $\rho>0$.

The most general result of this type which can be obtained as a simple consequence of our theorem can be stated as follows:

Let $A$ be an open $n$-dimensional set whose closure $\bar{A}$ has the property that for any $x \in \bar{A}$ and a fixed $c>0$ at least one of the cubes $C(x ; c)$ is completely contained in $\bar{A}$. If $f$ and its mixed derivatives $D^{p} f, p \in P$, are continuous in $\bar{A}$, then for any $x \in \bar{A}$ we have

$$
M(f(x)) \leqq \sum_{p \in P}\left(1+\frac{1}{c}\right)^{n-|p|} \int_{A} M\left(2^{n-|p|} D^{p} f(t)\right) d t .
$$

If $M(u)=u^{2}$, this result is a variant of an inequality of S. L. Sobolev (see [3, p. 243]). However, in Sobolev's inequality all derivatives of $f$ of order $m<n / 2$ appear on the right-hand side while here only the mixed derivatives are used.

To prove the inequality (3) let $J_{k}$ be the projection of the cube $C(x ; \rho)$ on the $x_{k}$-axis, $k=1, \cdots, n$, and let $I_{k}[f], k=1, \cdots, n$, be defined as follows:

$$
I_{k}[f]=\int_{J_{k}} \cdots \int_{J_{n}} M\left(f\left(x_{1}, \cdots, x_{k-1}, t_{k}, \cdots, t_{n}\right)\right) d t_{k} \cdots d t_{n} .
$$

Since $C(x ; \rho)=J_{1} \times \cdots \times J_{n}$ we have 


$$
I_{1}[f]=\int_{C(x ; \rho)} M(f(t)) d t .
$$

Finally let $P_{k}, k=1, \cdots, n+1$, be the following subset of $P$ :

$$
P_{k}=\left\{p \in P: p=\left(p_{1}, \cdots, p_{k-1}, 0, \cdots, 0\right)\right\} .
$$

Thus, $D^{p} f, p \in P_{k}$, is a mixed derivative of $f$ with respect to some of the first $k-1$ variables only.

We shall prove first the inequality

$$
I_{k}[f] \leqq \sum_{p \in P_{k}}\left(1+\frac{1}{\rho}\right)^{k-1-|p|} I_{1}\left[2^{k-1-|p|} D^{p} f\right]
$$

for all $k=1, \cdots, n$.

The inequality (5) is obviously true for $k=1$. Suppose that it is true for some $1 \leqq k<n$. We have then by the mean value theorem

$$
\begin{aligned}
\frac{1}{\rho} \int_{J_{k}} M\left(f\left(x_{1}, \cdots, x_{k-1}, t_{k}, \cdots, t_{n}\right)\right) d t_{k} & \\
& =M\left(f\left(x_{1}, \cdots, x_{k-1}, \theta_{k}, t_{k+1}, \cdots, t_{n}\right)\right),
\end{aligned}
$$

where $\theta_{k}=\theta_{k}\left(x_{1}, \cdots, x_{k-1}, t_{k+1}, \cdots, t_{n}\right) \in J_{k}$ and it follows that

$I_{k+1}[f]$

$$
\begin{aligned}
= & \int_{J_{k+1}} \cdots \int_{J_{n}} \int_{\theta_{k}}^{x_{k}}\left(\partial / \partial t_{k}\right) M\left(f\left(x_{1}, \cdots, x_{k-1}, t_{k}, \cdots, t_{n}\right)\right) d t_{k} \cdots d t_{n} \\
& +\frac{1}{\rho} \int_{J_{k+1}} \cdots \int_{J_{n}} \int_{J_{k}} M\left(f\left(x_{1}, \cdots, x_{k-1}, t_{k}, \cdots, t_{n}\right)\right) d t_{k} \cdots d t_{n} .
\end{aligned}
$$

Using the inequality (1) and the monotonicity of $M$ we obtain

$$
I_{k+1}[f] \leqq I_{k}\left[f_{k}^{\prime}\right]+\left(1+\frac{1}{\rho}\right) I_{k}[2 f] .
$$

Applying the inequality (5) to the right-hand side of this inequality we get

$$
\begin{aligned}
I_{k+1}[f] \leqq & \sum_{p \in P_{k}}\left(1+\frac{1}{\rho}\right)^{k-1-|p|} I_{1}\left[2^{k-1-|p|} D^{p} f_{k}^{\prime}\right] \\
& +\sum_{p \in P_{k}}\left(1+\frac{1}{\rho}\right)^{k-|p|} I_{1}\left[2^{k-|p|} D^{p} f\right] \\
\leqq & \sum_{q \in P_{k+1}}\left(1+\frac{1}{\rho}\right)^{k-|q|} I_{1}\left[2^{k-|q|} D^{q} f\right]
\end{aligned}
$$


since for any $p \in P_{k}$ there is a unique $q \in P_{k+1}$ with $|q|=|p|+1$ such that $D^{p} f_{k}^{\prime}=D^{q} f$ and for any $p \in P_{k}$ there is a unique $q \in P_{k+1}$ with $|q|=|p|$ such that $D^{p} f=D^{q} f$. Thus we have proved the inequality (5) by induction for all $k=1, \cdots, n$.

Finally, from

$$
\begin{aligned}
M(f(x))= & \int_{\theta_{n}}^{x_{n}}\left(\partial / \partial t_{n}\right) M\left(f\left(x_{1}, \cdots, x_{n-1}, t_{n}\right)\right) d t_{n} \\
& +\frac{1}{\rho} \int_{J_{n}} M\left(f\left(x_{1}, \cdots, x_{n-1}, t_{n}\right)\right) d t_{n}
\end{aligned}
$$

follows that

$$
M(f(x)) \leqq I_{n}\left[f_{n}^{\prime}\right]+\left(1+\frac{1}{\rho}\right) I_{n}[2 f] .
$$

Applying (5) with $k=n$ and using the same argument as before we obtain the inequality (3).

4. We mention finally two theorems from the theory of distributions which follow immediately from the inequality (4). These theorems were proved in [4] indirectly, the proofs in [4] being much more laborious than the present one (see [4, 2.1 (a) and (b), 3.11 and 3.1]).

Let $D^{p} f, p \in P$, be continuous and belong to $L_{M}^{*}(E)$. Then $f$ is bounded in $E$ and $f(x) \rightarrow 0(|x| \rightarrow \infty)$.

Let $D^{p} f_{i}, p \in P, i=1,2, \cdots$, be continuous and belong to $L_{M}^{*}(E)$ and let $\left\|D^{p} f_{i}\right\|_{M \rightarrow 0}(i \rightarrow \infty)$. Then the functions $f_{i}, i=1,2, \cdots$, are uniformly bounded in $E$ and $f_{i}(x) \rightarrow 0(|x| \rightarrow \infty)$ uniformly with respect to $i$.

\section{REFERENCES}

1. M. A. Krasnosel'skiY and Ja. B. RutickiY, Convex functions and Orlicz spaces, Fizmatgiz, Moscow, 1958.

2. L. Hörmander, Estimates for translation invariant operators in $L^{p}$-spaces, Acta Math. 104 (1960), 93-140.

3. A. Friedman, Generalized functions and partial differential equations, PrenticeHall, Englewood Cliffs, N. J., 1963.

4. J. Musielak, On some spaces of functions and distributions. I. Spaces $D_{M}$ and $D_{M}^{\prime}$, Studia Math. 21 (1961/1962), 195-202.

UNIVERSITY OF NOTRE DAME AND

University of Poznan, Poznan, Poland 